\title{
Study of the Effect of Imaginary Imagery (Imaginary Color, Imaginary Figure, Imaginary Space Building and Fantasy in Characterization) on the Characterization of Children
}

\author{
Iran Naseri Sisakht*, Ashkan Pourlashkary \\ Farhangian University, Yasuj, Iran \\ Email: *inaseri35@gmail.com
}

How to cite this paper: Sisakht, I. N., \& Pourlashkary, A. (2018). Study of the Effect of Imaginary Imagery (Imaginary Color, Imaginary Figure, Imaginary Space Building and Fantasy in Characterization) on the Characterization of Children. Advances in Literary Study, 6, 161-171.

https://doi.org/10.4236/als.2018.64014

Received: November 15, 2017

Accepted: August 18, 2018

Published: August 21, 2018

Copyright $\odot 2018$ by authors and Scientific Research Publishing Inc. This work is licensed under the Creative Commons Attribution International License (CC BY 4.0).

http://creativecommons.org/licenses/by/4.0/

\begin{abstract}
Provided introduction is related to imagination in children's story books. Reviewing the books of this course, in addition to introducing the author's styles, leads to more knowledge about the content of these books. The imaginary imagery as the main gem of these books is considered as one of the most important artistic criteria. Types of colors, shapes and characteristics are the most striking forms of imagination in this work. Applying live metaphors and exquisite combinations which appear on the vapor of imagery and the remarkable frequency of ecosystemism can be seen in these works. Children build the foundations of the future civilization; therefore, in order to understand and promote the culture of our country, the study of the effect of imagery in the works of Iranian illustrators associated with the stories of Iranian writers is considered as the research goal. Another goal of this study was to identify the elements that illustrators have been inclined to use to make more visual imagery over the past decade. The methodology of this research is historical and descriptive-analytical, and the collection of materials has been done through library and internet studies. The results of the review of books and images and examples from the age group " $\mathrm{B}$ and C" books by the Children's and Adolescent Intellectual Development Center indicate that the use of fictional imagery in the illustrations of the last decade has been growing and more illustrators have taken fantastic elements in creating the story space. The most commonly used fictional imagery is imaginary space, and the smallest is imaginative characters.
\end{abstract}

\section{Keywords}

Imaginary Imagery, Children's Story Illustration, Iran's Last Decade 


\section{Introduction}

Imagination or imagination for some is intuition and for others is the interference of man in the universe. The fantasy world creates a vast arena in the writer's mindset, leading to creativity, awakening, artistic expression, and discovery and intuition. As they say, "Dream is both a child of attitude and affection", and it is the task of embodying the emotional and emotional content of the writer's experience. The discovery of the basic imagery of the artist and the visualization of clusters puts the key to enter the world within the artist in our hands (Jalali, 2014). This interference, which begins first from the mind, can be expressed in various forms in art, literature, life and science. From the obtained works, it can be seen that Iranian art and literature have well-used theological and mystical views on imagination. Works like the mirror reflects another world. The forms of imagination are in the form of a science of expression and sometimes meanings and even a profound newness and rarely, in the works of the ancients, the elements of the imaginary construct can be derived from the combinations and associations or the environment from which they originated. In general, it can be said that the forms of the old imagination are limited in certain forms and limit their work in the analogous and permissive domain of metamorphism (Arjmandi, 2015). Literature, music, painting and architecture each reflect their own style and single style. Of course, the ancient times in the books of imaginative literature have also been considered as an opacity, which consists of two meanings, the meaning of the truth and the meaning permitted is allowed, and sometimes it constrained imagination in the concept of one of the less famous and worthless industries of old literature. Contemporary action is between the "image" and "affection", and they believe that they must have any image, affection and salinity. What makes sense of human imagination against perceptions is a fantasy that is the original essence and constant element of the poem and therefore primitive humans have generally been poets, because they are amazed in front of any normal and natural phenomenon, such as lightning, the fall of the leaves, but today, only a few people are awakening and have talent of awakening, and at moments, and just in the same triggers have become more frequent. What poets perceive to ordinary people are the first awakening and secondary experience and knowledge of his/her discovery. In fact, the first person who perceives an element of nature with another element, is a poet who, by speaking of this awareness of perception, actually shows that he is awake and that this awakening has been a direct and immediate concern and this awakening is not due to the will of the poet that is a spiritual event that is unconsciously reflected in the poet's poem. The reason for the variety of imagination in poetry works is due to the special experiences of each poet and the type of his/her images is more or less devoted to himself (Arjmandi, 2015). The subject of the present research is to study the imagination of imagination in the illustration of Iranian stories. Since the imagination has been the topic of Iranian mystical wisdom and tradition, recognizing and studying its position in contemporary art and contempo- 
rary arts, such as illustration, is very important. Today's painters are working in various fields and in the last few years even the design of the sign is also done by illustration. All factors, such as the ability of the fan and the power of design, and the tool used to create an illustration effect they complete the stages of an artistic creation in the time that reveal the hidden essence that comes from the illustrator's imagination. It can be said that this essence is the same imagery. Human is a creature that sometimes enjoys more of a fantasy because the reality of everything is beautiful in the ambiguity of imagination. As for many centuries, the Moon was beautiful and the light of the sky, and when it came to it, said: "loneliness, dryness and futility".

The reasons for choosing the topic are: The importance of illustrating the child's book in cultivating the children's minds and beliefs, as a result, the study of visual elements in the illustration of a book fluttering the child's wings and creativity flourishes. One of the main goals of this research is that the researcher in his/her similes goes beyond the realm of reality in order to increase his/her ability to create imagination and transplant images. Illustrator, by exposing an image of his/her fantasy world filled with his/her particular imaginary shapes, creates a passageway in which passengers with going on it, identify the meaning and carries the image. An expression that comes with imaginary imagery will be considered in addition to the apparent meaning. In addition to these, the illustrator can make a tangible expression of the intangible subject by appealing to imagery. So, the aim of identify the factors that influence the imagination is making the image of the imagery which transforms it into a magic and outstanding world and the audience will also grow as he encounters a pictorial text of his/her imagination and creativity. The questions to be asked are as follows: Is there any connection between the distributions of all types of book imagery? Are the imaginary imagery in the illustrations only rooted in the text of the book? In the following, we refer to a variety of imaginary imagery:

\section{Color in the Image Imagination}

Color is a visual element that puts a variety of feelings on the viewer and as we know, the meaning of many colors is derived from natural phenomena. The colors of the image affect the child's imagination rather than the words of the text. The presence of color in the image of children's credibility greatly increases and brings him closer to the elements of the image (Akrami, 2015). Painting gives a great opportunity to illustrate the imagination and imagination of the dazzling and delicate spaces of the viewer. It is difficult to imagine the lack of color in childish images. Magic color display next to each other such as street fireworks sparkles attract children attention and provides them with an opportunity to surf in the dreamland. In general, in reviewing the books of the club in the last decade $54 \%$ of the fiction books of the children of the age group B and C, color considered as imaginary element of imagery. 


\section{Functional Role of Color}

The application of chromatic colors (white, black and gray) with chromatic colors and their combination creates a variety of visual effects. In the book "The Young Man and the Tricky Tailor" illustrated by Loffer (2014), this enjoyment of black color could be seen, which, along with blending with other colors, increases the visual courage and metaphor in the illustration.

The application of colors can add to the sensory space and create a dreamy, sleeping and developing state; like the use of a gentle and pleasant blue color in watercolor images of the book "One Piece of Good Soil of God" by Karimi (2011), that shows the wishes and conversations of the island with a sensual and poetic melancholy. Pleasant colors purple in the book of "The same purple sneakers" (by Jamali \& Ahmadi; 2015), feeling the need for a place to sleep and hear a story that awakens the characters in the book text included in the reader.

The metaphorical and symbolic language in its simplest or complex form, especially in the illustration of conceptual and thought-provoking books, is regarded by illustrators who emphasize semiotic elements (Akrami, 2015). The use of red in the first frame of the bang-bang book by Mojaveri (2012) expresses the illustrator's efforts to create attention and risk alert in conjunction with a written voice. The color here is completely regarded as a visual and symbolic metaphor, in fact, since the red color represents blood and the weapon's function is also bleeding, and the child can easily understand the relationship between them (Figure 1).

\section{Imaginary Spatialization}

Spatialization can be one of the elements that helps the child to better understand and believe the hero of the story. Moving the location of the hero changes the space, and the storytelling events go to the next step. "The most practical form of spatialization of the hero or heroes of the story is in the center of the field where the momentary event of the image occurred in the background. In this way the hero of the story is at the center of attention and has taken a

\section{Bang}

Bang...!

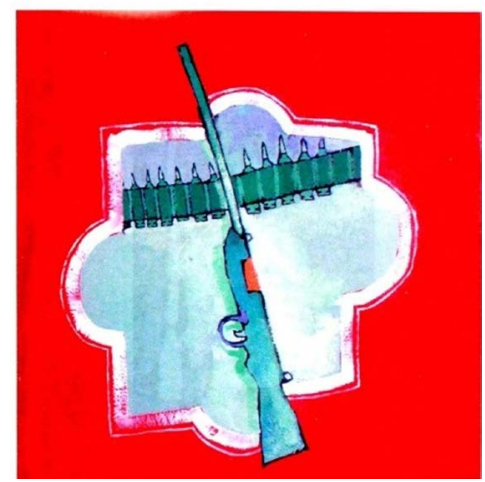

Figure 1. Take advantage of the symbolic and metaphorical effects of color in Bang Bang. 
descriptive space around him" (Akrami, 2014; Sajjadi, 2013). The purpose of imaginary space is to make the spatial and temporal situations more tangible and express the imaginary sense in the story to the audience to have a better understanding of the characters and actions of the story. "In fiction books, as in other literary works, space is used to determine the location of the time and create space of a story and to create an appropriate climate" (Loffler, 2014).

Away from the bustle irrelevant of space elements, next to the black or dark night in create space directs the child to silence and obscurity in the text tells the fantasy and the world of dreams. In The book of Merciful Moon with the Illustration of Mehrvarz (2012), we see such enjoyment of dark spaces, which beside the privacy simplicity and clarity of forms are very effective on induction of children's fantasies and childhood dreams (Figure 2).

As we know, in visual spatial we faced with two categories of spaces, inner space and outer space. Inner spaces such as the inner space of the house or a room with the equipment arranged there and in outer spatial, illiterator with more freedom illiterator with more freedom placed the nature of cities or villages or free nature in the background. In children's stories, the nature is the most visible form of space. For example, toys on the shelf of the children's room or the color of their room indicate inner space and a window in their room with a meadow background, considered as outer space.

\section{Imagination in Personification}

Characters make up structure of the story. Human, vegetarian, animal and even objects characters, may create fantastic and fantastic happenings in a story. $\mathrm{Hu}$ man and animal characters are among the most important characters in children's tales. Characters are sometimes realistic and constructive designs which can display scientific and historical characters and sometimes characters there are for creating imagination in pictorial.
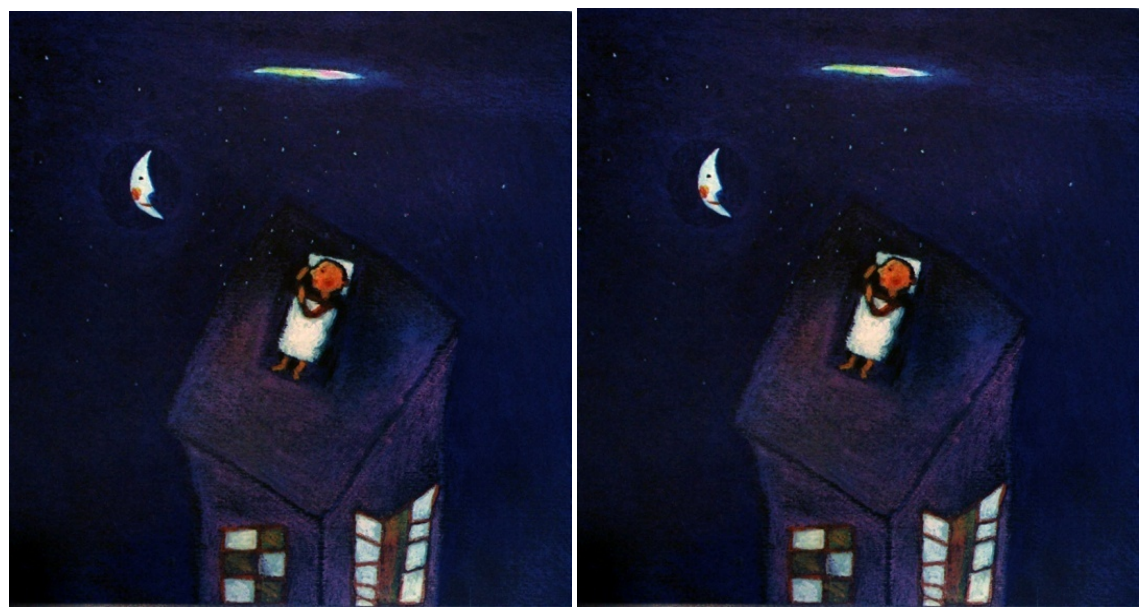

Figure 2. Use the layout of the elements of the image next to deep blue and purple colors to create a dreamlike and imaginative space in the book of Merciful Moon (Anthonyadis, 2015). 
In the review of books according to table, Injection of imagination by personification in the illustration in the last decade, only $42 \%$ of the total published book appropriated to itself.

\section{Anthropomorphism}

Sometimes the induction of imaginary space in the story can also be done by anthropomorphism "Humanism is a phenomenon in the writings and a picture of human actions in the form of speaking and behaviors of human beings and in the tales and legends of children has a widespread role. Give human personality to plants and objects has a lot of diversity in the pictograms (Ebrahimi Dinani, 2012; Ahmadpour, 2013)" (Figure 3).

In parts of the book, "Das, Das and Das, Nokhod and Ada," with illustration of Mohammadi (2014), somewhat anthropomorphism can be seen. Animals in shapes and behaviors designed similar to humans. Such as chicken and pigs are wearing clothes similar to humans and with a gesture that is special for humans, they are walking and by this way, image stimulates the audience's imagination. In fact, it can be visualized in terms of visual imagery in the category of visual metaphors.

\section{Illustrator Mental Characters}

Today's illustrators, in addition to characterizing myths, in characterizing real stories, like fiction stories, they enter abstraction and this is in the opposite direction to the illustration of previous decades which displayed the most fantastic legends in the form of realistic illustrations.

Also in myth, sometimes function of animal character is similar with human character. It can be said that characterization is directly related to the style and image of the illustrator and comes with a mixture of sensory elements in the "I".
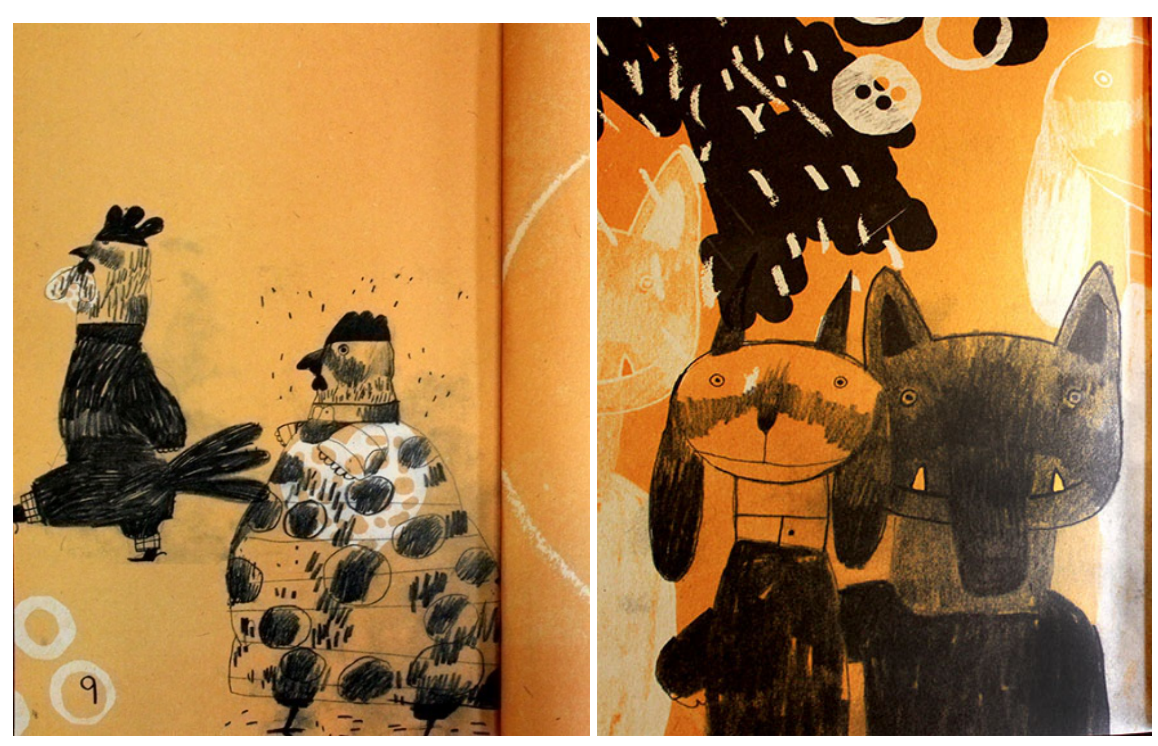

Figure 3. Role of anthropomorphism in imaginative character (Shafiei Kotkani, 2013). 
If we compare it, before the presence of abstraction in the illustrations of the 1960s and 70s in Iran, image characters in most cases it combines the features of Iranian painting and realism which is seen in children's books from other countries of the world. Such as book of "Carpet flowers" by Zabeti Jahromi (2014) that illustrated in 1980 (Figure 4).

As abstraction and imagination develop in imagery, the escape from reality of personification was also wider and new possibilities between the fantasy and inner feel of the hero of the story was created in connection with the author's style and angle of view. So that it can be seen from the overview which is in today's illustration of children's books in Iran, there is less realization of the image characters. When the fantasy and inner feel of the hero of the story reflects on his/her face and his/her actions which has oriented mode (expression), is a worthwhile feature that makes the book's personality viable for the child (Sheikholeslami, 2011; Mohsenian, 2015).

\section{Findings}

Table $1 \&$ Table 2 is based on the statistical population of the fictional story in

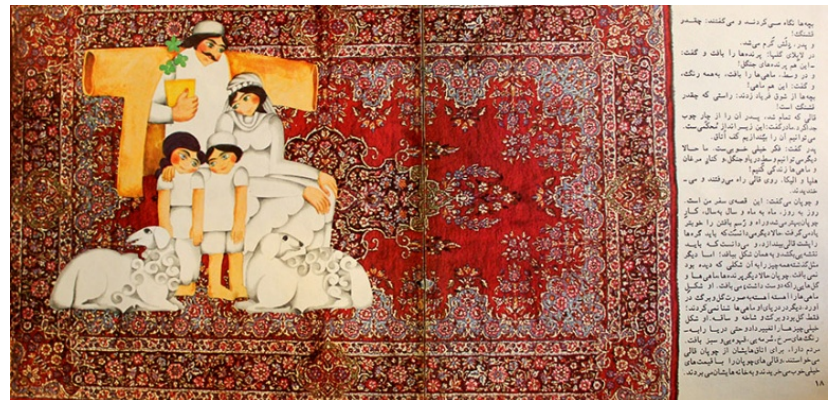

Figure 4. An example of characterization in the 60's, book of “Carpet flowers" (Ebrahimi Dinani, 2012).

Table 1. Review of the number of books with imaginary imagery in the last decade (Mehrors, 2014).

\begin{tabular}{lccc}
\hline Year & $\begin{array}{c}\text { The number of published } \\
\text { books by the club }\end{array}$ & $\begin{array}{c}\text { The number of story books in the } \\
\text { age group B and C }\end{array}$ & $\begin{array}{c}\text { Book with Imaginary } \\
\text { imagery }\end{array}$ \\
\hline 2000 & 55 & 9 & 1 \\
2001 & 55 & 13 & 4 \\
2002 & 68 & 10 & 3 \\
2004 & 34 & 6 & 1 \\
2005 & 100 & 17 & 4 \\
2006 & 102 & 9 & 4 \\
2001 & 73 & 3 & 5 \\
2009 & 53 & 4 & 2 \\
2011 & 29 & 6 & 6 \\
2013 & 71 & 5 & 4 \\
2015 & 69 & 8 & 6 \\
2016 & 73 & & 4 \\
\hline
\end{tabular}


Table 2. Table of the classification of types of imaginary imagery in the reviewed books (Mehrors, 2014).

\begin{tabular}{|c|c|c|c|c|c|}
\hline Row & Book Title & Imaginary Color & Imaginary Figure & Imaginary Space & Imagination in Characterizations \\
\hline 1 & Afsane Kachal-E-Kaftarbaz & * & * & * & - \\
\hline 2 & That Above This Low & - & * & * & * \\
\hline 3 & Ay Ghese Ghese Ghese & * & * & * & * \\
\hline 4 & Faith Multiplied By 81 & * & - & * & - \\
\hline 5 & This Is Also A Fish Money & - & * & * & - \\
\hline 6 & Kid All & - & * & * & * \\
\hline 7 & The Biggest Girl Of The Universe & * & * & * & * \\
\hline 8 & Bang Bang & * & * & * & * \\
\hline 9 & Tan Pich & * & * & * & - \\
\hline 10 & Birth Of A Butterfly & - & * & * & $* *$ \\
\hline 11 & The Magic Of The Town Hall & - & - & * & - \\
\hline 12 & Sunshine Girl And Black Demon & * & * & * & * \\
\hline 13 & Das -O- Das -O- Das, Nokhod -O- Adas & - & * & - & * \\
\hline 14 & Ali And The Prophet & - & * & * & - \\
\hline 15 & Eleventh Step & - & * & - & * \\
\hline 16 & Story Of The Red Lamb And The Sad Prince & * & * & * & - \\
\hline 17 & Story Of Panirak & * & * & * & * \\
\hline 18 & Story Of Koti Koti & - & * & - & * \\
\hline 19 & Story Of Phil And Cup & - & - & * & - \\
\hline 20 & $\begin{array}{l}\text { Lost (Illustrated By Mohammad } \\
\text { Ali Bani Asadi) }\end{array}$ & - & - & * & - \\
\hline 21 & Lost (Illustrated By Sodabeh Shiran) & - & * & * & - \\
\hline 22 & Lolo Pashmalo, Kerme Kocholo & * & * & * & * \\
\hline 23 & My Moon, Our Month & * & * & * & - \\
\hline 24 & Gentle Month & * & * & * & - \\
\hline 25 & Scarecrow And Mrs. Spring & * & * & * & * \\
\hline 26 & Like Everyone, But Like No One & - & - & * & - \\
\hline 27 & Young Man And Cunning Tailor & * & * & * & - \\
\hline 28 & Me And An Empty Pot & - & * & * & - \\
\hline 29 & Kind As Christ & * & * & * & - \\
\hline 30 & I'm Going To Nest For The Worms & * & * & * & - \\
\hline 31 & The Same Purple Shoes Lame & * & * & * & - \\
\hline 32 & One Piece Of Good Soil Of God & * & * & * & - \\
\hline \multirow[t]{2}{*}{33} & One Was & - & - & * & * \\
\hline & & 18 & 27 & 30 & 14 \\
\hline
\end{tabular}

* is positive and - is negative. 
the story books (Iranian author and illustrator) in the age group B and C, which has been published by the Intellectual Breeding Center for Intellectual Development of Children and Adolescents in the last decade. A total of 640 titles have been published in the last decade, of which only 33 books were created in the age group of the target audience and were created by Iranian illustrators with more emphasis on imaginative imagery.

\section{Conclusions}

Based on the searches performed in the target population, imaginary imagery in the illustrations of the storytelling books of the Sunni B and C children was published by the Iranian author and illustrator by Kanun and in the 2010s, significant results were obtained. In the early 1990s, about $11 \%$ of the books published in this age group created their own design based on imaginary forms, whereas it increased to $66 \%$ in the year 87 and peaked $100 \%$ in the years 2009 and 2010 . There are many reasons for this increase in values. In the category of fantasy imagery according to the tables presented in Chapter IV, the use of imaginary spaces has the highest rate which is equivalent to $90 \%$ of the books reviewed in the past decade. Contrary to imagination, the use of illustrators of imaginary personalities includes the lowest amount, which is $42 \%$. Overall, A total of 33 books that were investigated and in line with research hypotheses, illustrator in addition to the occasional use of literary fiction, the illustrator transcends the foot of the text and the imagery imaginary forms are based on the author's mentality and his/her creativity in line with the story. The free expressions of the illustrators of the story have led to the creation of fantasy elements and this means that the illustrator is not enclosed in the text. With the rise of communication, the holding of numerous illustrating festivals and academic studies that conducted in recent years in the field of illustration, the influence of our illustrators on the effects of non-Iranian works, especially the West, has increased. Illustration styles are in fact the same drawing styles. Modern painting began with the focus of human mind and imagination. In the last decade in Iran there are more tendencies to use modern styles in imaging that is related to the impact of modern styles on illustration and also the direct impact of styles on illustration is not seen. In most works there was a combination of modern painting styles, which is taking shape under the same influence of modernity and postmodernity and it can be due to the fact that in recent years attention has been paid to imagination within the framework of the known styles of Iranian illustration. It's better to have fun and inspire curiosity for imaginary pictures attracting the child's attention. It is also necessary to activate the child's imagination and help him to increase his mental ability and explain his feelings. It's better to imagine imagery connected with all aspects of the child's personality and does not abuse his childish needs. Images should take the child's inner needs seriously and strengthen his confidence in himself and in his future. Awareness of the child about the world in which he lives, nurturing habits and creating self-esteem and the sense 
of independence and creativity development are one of the most important goals that children are looking for in pictures. Literature promotes children's emotional development in many ways. Sci-fi imagery, it prompts them to reflect on the future. New realistic stories of children increase their curious about the recognition of human beings and their relationship with the environment. With the help of literature the child learns different ways of dealing of human the difficulties of his life and they learn from the characters of the story how to solve similar problems and understand the feelings of others. Inspired by the same experience children are sparked to writing or retelling images created and paid by your mind or run the show. Imagination helps the child expand his vocabulary. Children who play fantasies, listen to fictional stories, or someone who reads stories from the book for them, are more likely to understand the rest of the words and have a larger vocabulary. A fantasy about imaginary situations tells a person how to think in real life and solve his/her problems. In most families in the school and at home, these are the adults who decide on the child and even solve the problems. But in the imagination the child alone is allowed to decide that how to advance the story and eliminate and solve the problems. Therefore this imagination gradually will help it to solve real world problems.

Jensen (1980) says: "A human attains literary values in childhood and its profound effects appear in adulthood". Reading and familiarizing with literature has different effects on children, as Loffler (2014) says, "literature contributes to children's emotional development. The contribution of literature is the appearance of feelings and emotions and to give a picture of these feelings and identify it". Children respond to what they see. The reactions of children to literature are influenced by the advent of different factors and affect the linguistic, cognitive, social and moral development of children on how they are taken from an image. Beliefs, expectations, reading skills, economic status, social status, gender, individual style of the reader interact with him and his effectiveness. Typically, literary texts have different narrative structures from relatively simple in folk tales to nested, articulate and long designs in a novel with intricate themes, annotated character and complex language. Each type of literature has a unique feature and a different function. Also, colors of the image affect the child's imagination rather than the words so that, green means peace and tranquility, red means hostility and bloodshed or white means clearity and purity Different types of books allow children to understand and react to personal motivations and understand motivations that affect others and react to them. The hope is that this research has been able to provide valuable results to the writer and the researchers, and has an effective role in fiction.

\section{Proposal for Researchers}

Researchers can use this research to explore other factors that have affected children's book illustrations in recent years. The study of the effect of a story alone on the use of an illustrator of imaginary imagery can also be the subject of 
further research and a clear path to understand more Iranian illustrators and illustrators in order to grow the culture, art and literature of this border. It is also suggested that the impact of imaginary shapes on the incidence of creativity and the development of a child's personality be discussed.

\section{Research Limitation}

Interviews with children in different age groups to understand right effect of colors and shapes on their viewpoints.

\section{References}

Ahmadpour, N. (2013). A Study of Imaginary Imagery in Writing and Images of Three Works by Hoda Haddadi, the Book of the Month of Child and Adolescence, 188, 89 to 96.

Akrami, J. (2014). Child and Image (Volume 2). Tehran: Soroush.

Anthonyadis, A. C. (2015). Architectural Architecture, Architecture, Ahmad Reza I (Volume 1). Tehran: Soroush.

Arjmandi, M. (2015). Mental Image and Cinematography. Tehran: Farabi Foundation.

Ebrahimi Dinani, Gh. (2012). Fictitious Claims and Art, Quarterly Fantasy, 2nd, 6th to 11 th.

Jalali, Gh. (2014). Cinema from the Viewpoint of Thought (Vol. 1, 1st Printing). Tehran: Art Area.

Jamali, Sh. B., \& Ahmadi, H. (2015). Alamlnfs from the Point of View of Islamic Scholars and Its Adaptation to New Psychology. Tehran: Allameh.

Jensen, E. (1980) Art History. Translated by Reza Marzban, Tehran, in Collaboration with the Islamic Revolution Publications and Education Organization (Formerly Franklin).

Karimi, A. (2011). The Extent of Imagination and Reality in Myths. Optional, 11, 7-27.

Loffler, D. (2014). Ramsey's Afsaneh Language, Translation of Jalal Sattari. Tehran: Toos.

Mehrors, Z. (2014). What Is Myth? What Is Epic? Teacher's Growth, 139th, from 15 to 17.

Mehrvarz, M. (2012). Imagination in Illustration of More Charm. Moon of Child and Adolescents, 183, 83-87.

Mohammadi, M. (2014). Methodology of Criticism of Children's Literature. Tehran: Soroush.

Mohsenian, R. M. (2015). Communication (7th Printing). Tehran: Soroush.

Mojaveri, A. M. (2012). Surveyor's Style and Surrealist Spaces in Illustrating Fictional Literature, the Book of the Child and Adolescent Month, 189, from 91 to 101.

Sajjadi, S. J. (2013). Islamic Culture (Volume 2). Tehran: Koomesh.

Shafiei Kotkani, M. R. (2013). The Scriptural in Farsi Poetry. Tehran: Aware.

Sheikholeslami, A. (2011). Imagination, Example and Jamal in Islamic Mysticism. Tehran: Art Academy of the Islamic Republic of Iran.

Zabeti Jahromi, A. (2014). Cinema and the Structure of Poetic Images in Shahnameh. Tehran: Book Publishing. 